\title{
Individual vs. Aggregate Preferences: The Case of a Small Fish in a Big Pond
}

\author{
Douglas W. Blackburn \\ Graduate School of Business Administration, Fordham University, New York, New York 10023, \\ blackburn@fordham.edu \\ Andrey D. Ukhov \\ School of Hotel Administration, Cornell University, Ithaca, New York 14853, andrey.ukhov@gmail.com
}

\begin{abstract}
$\mathrm{W}$ e study the relationship between the risk preferences of individuals and the risk preferences of the aggregate economy. To emphasize the vast differences that can occur between individual and market preferences brought about through aggregation, we assume an economy consisting entirely of risk seekers. We show that such individuals can lead to an aggregate economy that is risk averse. The converse is also true. An aggregate economy that exhibits risk aversion does not imply an economy of individual risk averters. An economy demanding a risk premium can be formed from individuals who do not demand such compensation. Understanding the relationship between the preferences of individuals and the preferences of the aggregate economy is crucial for understanding the connection between the behavioral finance literature, which focuses on individual preferences, and the asset-pricing literature, which focuses on aggregate prices. We discuss empirical implications of these results.
\end{abstract}

Key words: risk aversion; risk seeking; investor sentiment; risk premium

History: Received August 14, 2008; accepted May 13, 2012, by Wei Xiong, finance.

\section{Introduction}

The relation between risk preferences of individual agents in the economy and the attitude toward risk in the aggregate is of fundamental importance in financial economics. Ultimately, aggregate preferences determine the price of risk in the economy. Yet individual biases characterize deviations from canonical fully rational financial models. Equilibrium asset-pricing models begin by modeling the behavior of individuals through a utility function. Aggregation is the necessary bridge that spans the gap between individual preferences and the observed economic aggregates. In this paper, we demonstrate that the difference between individual preferences and aggregated preferences can be large. We show that the differences are attributed to perfect competition, the existence of budget constraints, and agent heterogeneity.

The asset-pricing literature has grown in two important directions. The first line of literature focuses on the aggregate market. Theoretical and empirical models are used to explain fundamental aggregate market characteristics such as expected returns and volatility. ${ }^{1}$ The second line of literature focuses on

\footnotetext{
${ }^{1}$ This direction of research has been challenged by a series of anomalies and puzzles that document the difficulties that pricing models have in explaining economic aggregates. See Shefrin (2005), Constantinides (2002), and Hirshleifer (2001) for a review.
}

the behavior of individuals-the building blocks of the economy. Individuals are traditionally assumed to be risk averse. In recent years, however, a large literature has developed arguing that individuals do not make decisions according to standard concave utility functions but instead are averse to losses, are overconfident, tend to under- or overreact to different news events, choose consumption based on habit, and exhibit other behavioral patterns. Many nonstandard utility functions have been hypothesized to better model individual behavior.

These two lines of thought are connected through aggregation, yet the aggregation of nonstandard utility functions has not been adequately researched. It is only by aggregating individual demands that we can determine how individual behavior impacts aggregate prices. Yet this is a critical gap in the literature. This paper makes several important statements regarding the relationship between the aggregate economy and the individuals supporting the economy.

First, we consider an economy comprised solely of risk-seeking agents, each facing a budget constraint. ${ }^{2}$ We choose such a challenging economy to accentuate the importance of aggregation and to emphasize the

\footnotetext{
${ }^{2}$ We do not believe or suggest that all individuals are actually risk seeking. We use this extreme assumption to contrast individual and aggregate behavior.
} 
large changes in preferences that are possible when aggregating. Because of the convex shape of the riskseeking utility function, risk-seeking agents will trade to hold infinite positions in a single good. A budget constraint is imposed to prevent such infinite holdings. We first show that homogeneous risk-seeking agents facing perfect competition and a budget constraint result in an aggregate economy that is risk neutral.

Second, we build on the first result by showing that agent heterogeneity can lead to an aggregate economy that is risk averse. When risk-seeking agents facing perfect competition and a budget constraint are distributed across a large number of wealth classes, the aggregate economy has convex and differentiable indifference curves, implying risk aversion in the aggregate. Although individuals are risk seeking, the aggregate market can demand a risk premium. This result suggests that it cannot be immediately assumed that the use of nonstandard utility functions will lead to an aggregate economy with similar nonstandard preferences.

Third, we disaggregate a representative agent economy to show that the connection between the observed aggregate behavior and individual behavior is not obvious. We prove that a given aggregate economy that demands a risk premium can be decomposed into an economy consisting only of risk-seeking individual agents. We cannot, therefore, conclude that individuals are risk averse simply by observing a risk premium in aggregate prices.

Our findings have important theoretical and empirical implications. With the growing number of anomalies and puzzles found in the aggregate data, researchers have turned to behavioral theories with the hypothesis that individual behavioral biases can help explain the observed aggregate data. Behavioral theories clearly lead to a better understanding of how individuals make decisions, but it is unclear how the individual behavior affects observed aggregate prices. Our results show that it is necessary to explicitly aggregate agents' preferences. In addition, we need to build models with rich market environments, understanding that the environment can distort the aggregate demands of individuals with behavioral biases.

Regarding empirical research, our results have implications for understanding and interpreting empirical puzzles observed in the aggregate data. Unusual utility functions have been proposed to explain individual behavior. However, the data most commonly used in financial research are aggregate. Care must be taken when making conclusions concerning aggregate data based on theories describing individual behavior. Furthermore, although it may be tempting to make statements concerning individual behavior based on aggregate data, the analysis in this paper shows that the relationship between groups and individuals is not obvious. As we discuss in more detail in $\$ 5$, studies based on individual behavioral data and studies based on controlled experiments where the researcher is able to observe individual behavior as well as the aggregate outcomes are necessary areas of research to help understand the link between the individual and the aggregate.

The remainder of this paper is organized as follows. In $\S 2$, we discuss the intuition behind our theoretical results, highlighting the importance of perfect competition and budget constraints. Our theoretical results are formally presented in $\S \S 3$ and 4 . We study the aggregation of convex utility functions in §3, beginning with the simplest case of identical agents and then proceeding to an economy of heterogeneous agents. In $\S 4$, we prove that every risk-averse aggregate economy can be obtained by aggregating riskseeking individual agents. We relate our results to the literature and discuss their empirical and theoretical implications in \$5. Concluding remarks are provided in $\S 6$. All proofs are in the appendix.

\section{Model Intuition}

Before proving our results formally as theorems, we discuss the economic intuition leading to our conclusions. Our claim is that perfect competition, market frictions, and agent heterogeneity, in addition to the shape of the utility function, all influence the allocation of resources that individuals hold. As a result, the observed aggregate preferences can be very different from the preferences of the individuals supporting the economy. In this section, we discuss how these various market forces affect aggregate preferences in the context of an economy consisting of agents with risk-seeking preferences.

Our fundamental approach centers on tracing the aggregate indifference curve. There are two goods, $X$ and $Y$, representing two sources of utility. We start with various initial supplies of the $X$ good. For each initial quantity of $X$, the quantity of the $Y$ good is determined so that (1) the aggregate quantity of $Y$ is minimized and (2) each individual agent receives an allocation so that he always remains on the same indifference curve. The first requirement establishes the efficient use of resources. When the quantity of the $Y$ good is minimized, no utility increasing coalition will exist. The second requirement ensures that the minimum quantity of $Y$ demanded by the economy is sufficient for each individual agent to always be on the same indifference curve, regardless of the initial supply of $X$. The aggregate indifference curve is, 
therefore, the plot of all aggregate quantities of the $X$ and $Y$ goods satisfying the two conditions. ${ }^{3}$

Risk averters (agents with standard concave utility) desire diversification. Their dislike for risk acts as an internal constraint, causing them to hold only the allocations with the appropriate risk-reward trade-off. The concave shape of their utility imposes internal discipline on agent behavior.

Risk-seeking agents are different because they do not possess such an internal constraint. Through trading, risk seekers want to infinitely short one good to infinitely long another. External constraints (market frictions), such as short-sale limitations, costly borrowing, and so on, can prevent the agent from achieving the desired infinite position. ${ }^{4}$ Risk seekers will make large trades until their constraints bind, that is, corner allocations. Perfect competition ensures that all (except at most one agent) will hold a corner allocation. Each individual agent's allocation is determined not only by the risk-seeking shape of the utility function but also by all binding external constraints. Likewise, aggregate preferences must also reflect all binding economic frictions (including competitiveness, heterogeneity, and trading limitations) and not merely reflect the shape of the utility function.

As a simple example to illustrate the intuition, consider a large economy of identical risk-seeking individuals with identical endowments across two states of nature. Agents' preferences across the two states are described by the time separable utility function $E\left[U\left(C_{1}\right)+U\left(C_{2}\right)\right]$, where $C=C_{1}=C_{2}$ is the aggregate consumption, $U(\cdot)$ is the agents' risk-seeking utility function, and $E[\cdot]$ denotes the expectation operator. Short selling consumption is not allowed. In this simple setting, prices of the two states are equal. Riskseeking agents seek to specialize in one good. Therefore, half of the population will enter a bet that pays 1 unit of consumption in state one and 0 in state two. The other half of the population will take the opposite bet. Interestingly, though each individual is risk seeking, the economy behaves as if it is risk neutral.

In our analysis, the risk-seeking shape of the utility function, perfect competition, budget constraints, and heterogeneity are all important. The risk-seeking shape provides a motive for trading. Perfect competition first creates the opportunity for trade, allowing the market to arrive at an efficient allocation of the limited resources; second, it causes each agent to be atomistic. Because markets must clear, at most one

\footnotetext{
${ }^{3}$ This is a standard approach to the efficiency problem (e.g., Atkeson and Lucas 1992).

${ }^{4}$ Such constraints are realistic. First, although we consider the noshort-sell constraint, any finite limit to short selling will have the desired effect. Second, there are limited resources, so holding infinite $X$ is impossible. Third, borrowing (short-selling) restrictions are observed in practice.
}

agent will be left holding some of both goods-an interior position. The one atomistic agent has negligible demand and therefore does not affect aggregate preferences (the proverbial small fish). ${ }^{5}$ The budget constraint is an external force preventing riskseeking agents from achieving their desired infinite positions. Heterogeneity allows a variety of rates at which agents will trade goods, creating the convex curvature (risk aversion) of the aggregate indifference curve. We now formally prove our conjectures.

\section{Convex Utility}

In a typical model, an agent solves the expected utility maximization problem,

$$
\max \mathbb{E}\left[u\left(C_{0}, \tilde{C}_{1}\right)\right],
$$

subject to a budget constraint. The utility of consumption is often assumed to be concave and additively separable:

$$
u\left(C_{0}, C_{1}\right)=U_{0}\left(C_{0}\right)+U_{1}\left(C_{1}\right)
$$

This is a convenient assumption mathematically because it implies an internal optimum. We follow this standard approach but with one change. We depart from the concavity (risk-aversion) assumption and instead assume that agents are risk seeking, having strictly convex utility functions. We choose such a challenging setting to illustrate the dramatic effects that competition, economic constraints, and trading across agents have on aggregate preferences.

There are two sources of utility in the economy, referred to as the " $X$ consumption good" and the " $Y$ consumption good." We denote aggregate quantities of the goods using uppercase boldface $\mathbf{X}$ and $\mathbf{Y}$; individual endowments are denoted by lowercase $x$ and $y$. The goods can be interpreted as the allocation at two different points of time or in two different states.

Each agent $i$ has a strictly risk-seeking utility function that corresponds to strictly concave indifference curves. By the implicit function theorem, we write each agent's utility function in terms of the indifference curves, $y(x)=\Psi(x ; k)$, where $k$ is the level of utility. We define $y_{\max }=\Psi(0 ; k)$ as the quantity of $Y$ so that the individual has utility $k$ when $x=0$. We define $x_{\max }=\Psi^{-1}(0 ; k)$ in a similar way. The notation $\mathbf{Y}_{\max }\left(\right.$ or $\mathbf{X}_{\max }$ ) refers to the aggregate quantity of $Y$ (or $X$ ) required so that all agents have utility of $k$ in the absence of $X$ (or $Y$ ). A consumption bundle that includes positive quantities of both $X$ and $Y$ is called

${ }^{5}$ This is a standard setting of perfect competition (Aumann 1964; Hellwig 1980, 1982). 
an interior allocation, and a consumption bundle characterized by zero units of either $Y$ or $X$ is called a corner allocation.

To model perfect competition, we follow Aumann (1964) by considering an infinite number of agents. ${ }^{6}$ We begin with $N$ agents and then allow $N$ to increase to infinity while holding the maximum possible quantities of $X$ and $Y, \mathbf{X}_{\max }$ and $\mathbf{Y}_{\max }$, constant. This creates a sequence of economies where the primary difference between elements in the sequence is the number of agents. As the number of agents increases, each agent's allocation becomes smaller. As described by Aumann (1964), an agent's allocation can be considered "infinitesimal" in the limit. Hence, in the limit, no individual agent has influence over the market. ${ }^{7}$

For each initial aggregate endowment of the $X$ good, $\mathbf{X} \in\left[0, \mathbf{X}_{\max }\right]$, an efficient aggregate bundle $(\mathbf{X}, \mathbf{Y})$ satisfies the following properties. First, all $\mathbf{X}$ must be held by some collection of agents. This is a market-clearing condition. Second, agents cannot hold negative quantities of either consumption good-no short selling of consumption. Third, the economy is endowed with the minimum aggregate quantity of the $Y$ good such that all agents maintain the same level of utility for all aggregate endowments of the $X$ good. The existence of a coalition that can increase their overall aggregate utility via trading contradicts the requirement of a minimum aggregate quantity of $Y$. Hence, the problem to be solved is

$$
\begin{aligned}
\min _{x_{1}, \ldots, x_{N}} \quad \mathbf{Y} & =\sum_{i=1}^{N} \Psi\left(x_{i} ; k_{i}\right) \\
\text { subject to } \quad \mathbf{X} & =\sum_{i=1}^{N} x_{i} \quad \text { and } \\
x_{i} & \geq 0, y_{i} \geq 0 \quad \text { for all } i .
\end{aligned}
$$

The no-short-sale constraint is an important assumption. Whereas risk-averse agents have strong preferences for smooth consumption, risk-seeking agents prefer volatile consumption achieved by infinitely shorting one good to hold an infinite quantity of the second good. Our no-short-sale restriction is reasonable because there are both finite natural and production resources preventing the existence of an infinite quantity of any good. It is important to note that our results will hold for any finite short-sale constraint by redefining the coordinate axis through a change of variables so that the constraint is redefined

\footnotetext{
${ }^{6}$ Hellwig $(1980,1982)$ argues that it is important to work within a competitive economy where the number of agents is infinite (in the limit). We construct a competitive economy similarly.

${ }^{7}$ An alternative approach is to consider a continuum of agents. Although this approach yields the same results, the intuition and discussion are clearer using a countable number of agents.
}

to be zero. What is important to our analysis is to rule out infinite borrowing, not borrowing per se.

The analysis progresses in a systematic way providing the set of conditions under which an economy of risk-seeking agents can become risk averse in the aggregate. We begin with the simplest case. An economy of homogeneous risk-seeking agents facing perfect competition and a budget constraint leads to a convex (linear) aggregate indifference curve. The agents are homogeneous, with identical utility functions and initial endowments. We then relax the homogeneity assumption by allowing agents to be heterogeneous in wealth. If, in addition to perfect competition and a budget constraint, the risk-seeking agents are divided into two wealth classes, then the aggregate indifference curve is strictly convex (piecewise linear) but not differentiable. As a final step, if the risk-seeking agents, facing both perfect competition and a budget constraint, are distributed across a large number of wealth classes, then the aggregate indifference curve is strictly convex and differentiable, corresponding to an aggregate risk-averse economy.

\subsection{Homogeneous Agents}

The analysis proceeds as follows. First, Proposition 1 describes each agent's demand for the two goods. We then aggregate individual demands to show in Proposition 2 that the aggregate indifference curve is linear. Homogeneous risk-seeking agents appear to be risk neutral in the aggregate.

Suppose only the $X$ good is available. By the assumption of homogeneity, each of the $N$ agents holds equal quantities of the $X$ good, $\mathbf{X} / N=x_{\max }=$ $\Psi^{-1}(0 ; k)$, and all agents are equally happy with utility $k$. Starting with only the $X$ good allows us to define the utility $k$ and $\boldsymbol{X}_{\max }$. For all $\mathbf{X} \in\left[0, \boldsymbol{X}_{\max }\right]$, the objective function in (1) is minimized with $k_{i}=k$ for all agents indexed by $i$.

When $\mathbf{X}<\mathbf{X}_{\max }, Y$ must be introduced into the economy for all agents to maintain a utility of $k$. The primary feature of the efficient allocation is that at most a single agent holds both the $X$ and $Y$. All other agents specialize in either $X$ or $Y$. To see that this allocation is efficient, suppose two agents hold allocations consisting of both $X$ and $Y$. By the concavity of the indifference curve, the agents trade with one another to increase the volatility of their consumption, resulting in an increase in utility. Trade continues until the budget constraint binds. The existence of such a coalition implies that the initial allocation was not efficient. We now state Proposition 1, which formally describes the efficient allocation of the $X$ and $Y$ goods.

Proposition 1 (Efficient Allocation for IdenTICAL Agents). Define $x_{\max }=\Psi^{-1}(0 ; k)$ and $y_{\max }=$ $\Psi(0 ; k)$. Let $k_{i}=k$ for $i=1,2, \ldots, N$. Any $\mathbf{X} \in\left[0, \mathbf{X}_{\max }\right]$ 
can be written as $\mathbf{X}=n x_{\max }+r$ for some $n=$ $0,1,2, \ldots, N$ and $r \in\left[0, x_{\max }\right)$. The solution to (1) is

$$
\left(x_{i}, y_{i}\right)= \begin{cases}\left(x_{\max }, 0\right) & \text { for } i=1 \text { to } n, n \geq 1, \\ (r, y(r)) & \text { for } i=n+1, \\ \left(0, y_{\max }\right) & \text { for } i=n+2 \text { to } N\end{cases}
$$

where $y(r)=\Psi(r ; k)$.

The aggregate demand for the $Y$ good is determined by summing all $Y$ allocations. There are $N-n$ agents who hold only the $Y$ good, and there is at most one agent who holds a positive quantity of both the $X$ and the $Y$ goods. The single agent holds the allocation $(r, y(r))$ where $r \in\left[0, x_{\max }\right)$ and $y(r) \in\left(0, y_{\max }\right]$. The aggregate demand $\mathbf{Y}$ as a function of $\mathbf{X}$ is

$$
\mathbf{Y}_{N}(\mathbf{X})=(N-n) y_{\max }+y(r) \quad \text { for } \mathbf{X} \in\left[0, \mathbf{X}_{\max }\right]
$$

We now formally show that an economy consisting of agents with concave indifference curves, under perfect competition and identical initial wealth endowments, has a convex (linear) indifference curve in the aggregate. Though individual agents are risk seeking, the aggregate economy behaves as if it is risk neutral. ${ }^{8}$

Proposition 2. Let $\mathbf{X}_{\max }$ and $\mathbf{Y}_{\max }$ be fixed quantities. As the number of agents $N$ approaches infinity, then $\mathbf{Y}_{N}(\mathbf{X})$ converges to $\mathbf{Y}(\mathbf{X})$ uniformly on $\left[\mathbf{0}, \mathbf{X}_{\max }\right]$, where

$$
\mathbf{Y}(\mathbf{X})=-\frac{\mathbf{Y}_{\max }}{\mathbf{X}_{\max }} \mathbf{X}+\mathbf{Y}_{\max }
$$

This result relies on the two assumptions of perfect competition and no infinite borrowing. Perfect competition reduces the influence of the single agent holding the interior allocation to zero, causing the aggregate demand to be determined by the agents holding corner allocations. Proposition 2 demonstrates that perfect competition and market frictions can cause aggregate preferences to be vastly different from the preferences of the individuals supporting the economy. An economy of risk-seeking individuals can be risk neutral.

\subsection{Heterogeneous Agents}

We take the analysis an additional step by providing conditions under which the aggregate indifference curve is strictly convex. In addition to a budget constraint and perfect competition, we now show that

\footnotetext{
${ }^{8}$ As an example, consider an exchange economy with two identical risk-seeking agents with the utility function $U\left(x_{i}, y_{i}\right)=x_{i}^{2}+y_{i}^{2}$ for $i=1,2$. There is no shorting. Because the example deviates from perfect competition, we consider only allocations associated with corners. Agents are equally happy with either the all $X$ corner allocation $\left(x_{i}, y_{i}\right)=(10,0)$ or the all $Y$ corner allocation $\left(x_{i}, y_{i}\right)=(0,10)$, leading to the three possible efficient aggregate quantities: $(\mathbf{X}, \mathbf{Y})=$ $(20,0),(10,10)$, and $(0,20)$. The three points form the linear aggregate indifference curve.
}

heterogeneity among risk-seeking agents is sufficient for the aggregate economy to appear risk averse. These are sufficient conditions to demonstrate that even if each agent is risk seeking, the aggregate preferences are consistent with risk aversion. The importance of modeling agent heterogeneity has become increasingly evident. ${ }^{9}$ We build on the results of the previous section by relaxing the assumption of investor homogeneity.

Investor heterogeneity can take many forms. In this analysis, we are specifically interested in each individual's trade-off between the $X$ and $Y$ goods. The previous analysis shows that the rate at which agents are willing to trade one corner allocation for the opposing corner allocation, $y_{\max } / x_{\max }$ is of particular significance. In this section, we allow agents to have differing rates of substitution.

Differences in substitution rates can arise in several ways. We can assume that agents have the same wealth and different risk-seeking utility functions. For example, for some agent $i$, the utility function can be of the form

$$
u_{i}(x, y)=\alpha_{i} x^{2}+\beta_{i} y^{2} \quad \text { with } \alpha_{i}, \beta_{i}>0 .
$$

In this case, $y_{i, \max } / x_{i, \max }=\alpha_{i}^{1 / 2} \beta_{i}^{-1 / 2}$, and hence the rate of substitution is dependent on each agent's $\alpha_{i}$ and $\beta_{i}$. Alternatively, we can model agents with identical utility functions but different levels of wealth. For example, agent $i$ 's utility function can be of the form

$$
u_{i}(x, y)=\alpha x^{2}+\beta y^{4} .
$$

Wealthier agents will be on higher indifference curves. Thus, if agent $i$ 's utility is $k_{i}$, then $y_{i, \max } / x_{i, \max }=$ $\alpha^{1 / 2}\left(k_{i} \beta\right)^{-1 / 4}$. The rate of substitution is a function of the agent's utility. Figure 1 illustrates the case where an agent with less wealth is willing to exchange a greater quantity of $Y$ for each unit of $X$ than the agent with greater wealth.

The logic of our argument presented in this section holds for either case so long as there is heterogeneity in the trade-off between $X$ and $Y$. For the purpose of our discussion, we use the language of wealth heterogeneity with the rate of substitution of the poor being greater (in absolute value and without loss of generality) than that of the rich. We assume that all agents have the same utility function and differ only in wealth. The intuition and results from the previous section will be used to argue and prove the results in this section.

\footnotetext{
${ }^{9}$ The distribution of wealth has been shown to affect the risk premium and risk-free rate (Gollier 2001), the allocation of resources (Esteban and Ray 2006), and economic growth (Foellmi and Zweimuller 2006). Risk-averse individuals who value status as represented by relative wealth can appear to have risk-taking preferences over certain regions of wealth (Gregory 1980, Robson 1992).
} 
Figure 1 Indifference Curves for Heterogeneous Risk-Seeking Agents

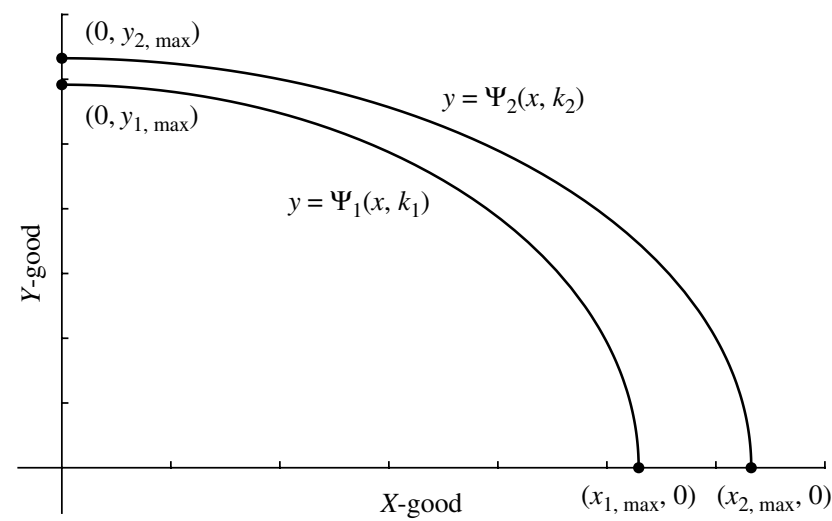

Notes. We plot the indifference curves, $y=\Psi_{i}\left(x, k_{i}\right)$, for two risk-seeking agents, $i=1,2$, with heterogeneous preferences. Two points are labeled for each agent. The point $\left(x_{i, \max }, 0\right)$ represents the allocation comprised of zero $Y$-good and the exact quantity of the $X$-good so that the agent is on the indifference curve $k_{i}$. Similarly, the point $\left(0, y_{i, \max }\right)$ represents the case such that the agent's allocation is comprised of the exact quantity of the $Y$-good for the agent to be on the indifference curve $k_{i}$ in the absence of the $X$-good. Preferences are such that $\left|y_{1, \max } / x_{1, \max }\right|>\left|y_{2, \max } / x_{2, \max }\right|$.

We first show that the aggregate economy's indifference curve is strictly convex but not differentiable with the introduction of two wealth classes. We then show that the aggregate indifference curve can be made strictly convex and differentiable with a sufficiently large number of wealth classes. In the limit, as the number of wealth classes increases to infinity, the aggregate indifference curve is smooth and convex (which corresponds to risk aversion).

Suppose there are $N_{1}+N_{2}$ agents divided into two wealth classes. There are $N_{1}$ type 1 agents (the poor) who have initial wealth less than the $N_{2}$ type 2 agents (the rich). We first consider the case where the economy contains only $\boldsymbol{X}_{\max }$ and none of the $Y$ good. Wealth is distributed so that the efficient allocation of $\mathbf{X}_{\max }$ between the two types causes the type 1 agents to be on the indifference curve $k_{1}$ and the type 2 agents to be on the $k_{2}$ indifference curve with $k_{1}<k_{2}$. Because agents within the same wealth class are homogeneous, all poor agents have identical allocations, and all rich agents have identical allocations. To trace the aggregate indifference curve of the economy, the minimum quantity of the $Y$ good is determined so that for each $\mathbf{X} \in\left[0, \boldsymbol{X}_{\max }\right]$, the poor agents remain on the $k_{1}$ indifference curve and the rich agents remain on the $k_{2}$ indifference curve.

As $N_{1}$ and $N_{2}$ increase to infinity, the existence of two different rates of substitution creates a kink in the aggregate indifference curve (Figure 2). The kink occurs when the aggregate supply of the $X$ good is $\mathbf{X}=N_{1} x_{1, \max }$. When $\mathbf{X}<N_{1} x_{1, \max }$, because the poor are willing to substitute more $Y$ for each unit of $X$ received (by assumption), then to minimize the aggregate quantity of the $Y$ good it is optimal for $\mathbf{X}$ to
Figure 2 Aggregate Indifference Curve with Two Agent Types

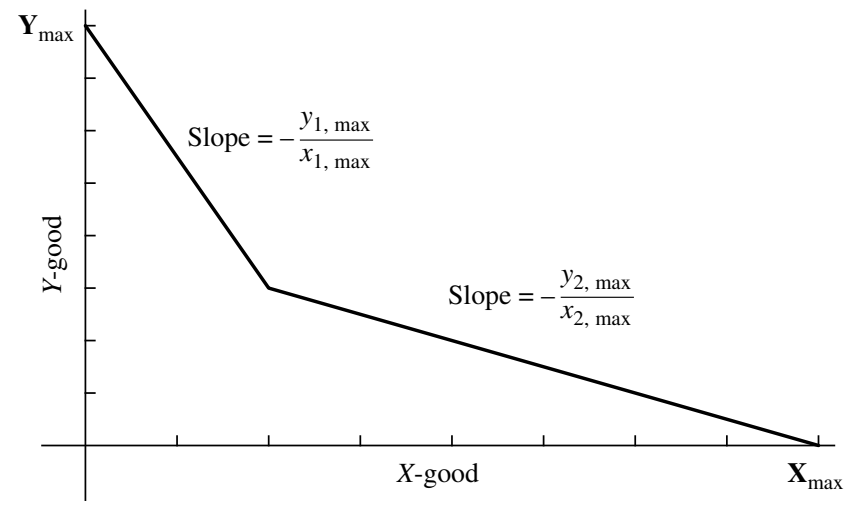

Notes. We plot the aggregate indifference curve in the limit as the number of risk-seeking agents in each of the two wealth classes increases to infinity. Agents fall into two groups such that $\left|y_{1, \max } / x_{1, \max }\right|>\left|y_{2, \max } / x_{2, \max }\right|$. The aggregate indifference curve is piecewise linear.

be held only by the poor. The economy-wide rate of substitution is then defined by the rate of substitution of the poor. However, when the aggregate quantity of $X$ is large, $\mathbf{X}>N_{1} x_{1, \max }$, there is enough of the $X$ good for all of the poor to hold their $X$ corner allocation. To clear the market, some rich agents must hold the remaining $X$ good. In this case, the economy-wide rate of substitution is defined by the rate of substitution of the rich. When $\mathbf{X}=N_{1} x_{1, \max }$ the rich and poor are perfectly separated, with all of the rich holding their $Y$ corner allocation and all of the poor holding their $X$ corner allocation, thus creating a kink in the aggregate indifference curve.

In proving our results, we follow the same fundamental approach to resource allocation as in the previous section. For each $\mathbf{X} \in\left[0, \mathbf{X}_{\max }\right]$, we minimize $\mathbf{Y}$ allocated to agents of both types so that the poor agents maintain their utility level $k_{1}$ and the rich agents maintain their utility level $k_{2}$. By the implicit function theorem, the equation $u(x, y)=k$ defines the implicit function $y=\Psi(x ; k)$. There are two constraints. First, all of the $X$ good must be allocated. Second, there are no short sales. We formally describe the allocation of resources in Proposition 3 and then prove that the aggregate indifference curve is piecewise linear (as in Figure 2) in Proposition 4.

Proposition 3. Let all agents have the same convex and differentiable utility function that is strictly increasing over all $x \geq 0$ and $y \geq 0$, but agents differ in initial wealth. The economy consists of $N_{1}$ type 1 agents (poor) and $\mathrm{N}_{2}$ type 2 agents (rich). The type 1 agents have utility $k_{1}$ and the type 2 agents have utility $k_{2}>k_{1}$ such that $\left|y_{1, \max } / x_{1, \max }\right|>\left|y_{2, \max } / x_{2, \max }\right|$, where $x_{h, \max }=$ $\Psi^{-1}\left(0 ; k_{h}\right)$ and $y_{h, \max }=\Psi\left(0 ; k_{h}\right)$ for $h=1,2$; we define $\mathbf{X}_{\max }=N_{1} x_{1, \max }+N_{2} x_{2, \max }$.

If $\mathbf{X} \in\left[0, N_{1} x_{1, \max }\right]$, then for $r_{1} \in\left[0, x_{1, \max }\right), y\left(r_{1}\right)=$ $\Psi\left(r_{1} ; k_{1}\right)$ and writing $\mathbf{X}=n x_{1, \max }+r_{1}$ for some integer 
value $n=0,1,2, \ldots, N_{1}$, the efficient allocation is

$$
\begin{gathered}
\left(x_{1, i}, y_{1, i}\right)= \begin{cases}\left(x_{1, \max }, 0\right) & \text { for } i=1 \text { to } n, n \geq 1, \\
\left(r_{1}, y\left(r_{1}\right)\right) & \text { for } i=n+1, \\
\left(0, y_{1, \max }\right) & \text { for } i=n+2 \text { to } N_{1} ;\end{cases} \\
\left(x_{2, j}, y_{2, j}\right)=\left(0, y_{2, \max }\right) \text { for } j=1 \text { to } N_{2} .
\end{gathered}
$$

If $\mathbf{X} \in\left(N_{1} x_{1, \max }, \mathbf{X}_{\max }\right]$, then for $r_{2} \in\left[0, x_{2, \max }\right), y\left(r_{2}\right)=$ $\Psi\left(r_{2} ; k_{2}\right)$ and writing $\mathbf{X}=N_{1} x_{1, \max }+n x_{2, \max }+r_{2}$ for some integer value $n=0,1,2, \ldots, N_{2}$, the efficient allocation is

$$
\begin{gathered}
\left(x_{1, i}, y_{1, i}\right)=\left(x_{1, \max }, 0\right) \text { for } i=1 \text { to } N_{1} ; \\
\left(x_{2, j}, y_{2, j}\right)= \begin{cases}\left(x_{2, \max }, 0\right) & \text { for } j=1 \text { to } n, n \geq 1, \\
\left(r_{2}, y\left(r_{2}\right)\right) & \text { for } j=n+1, \\
\left(0, y_{2, \max }\right) & \text { for } j=n+2 \text { to } N_{2} .\end{cases}
\end{gathered}
$$

The efficient allocation in Proposition 3 is very similar to the allocation described in Proposition 1. All but at most one agent specialize in either $X$ or $Y$. The remaining single agent may hold some of both goods. However, the single agent's influence on aggregate demand vanishes to zero as the number of agents in each wealth class increases to infinity. In the limit, aggregate demand is determined by those holding corner allocations. As a result, whereas Proposition 2 describes a linear aggregate indifference curve, the aggregate indifference curve described in Proposition 4 is piecewise linear and convex.

Proposition 4. Let the economy consist of $N_{1}$ type 1 agents (poor) and $N_{2}$ type 2 agents (rich). The type 1 agents have utility $k_{1}$ and the type 2 agents have utility $k_{2}>k_{1}$ such that $\left|y_{1, \max } / x_{1, \max }\right|>\left|y_{2, \max } / x_{2, \max }\right|$, where $x_{i, \max }=\Psi^{-1}\left(0 ; k_{i}\right)$ and $y_{i, \max }=\Psi\left(0 ; k_{i}\right)$ for $i=1,2$. For $\mathbf{X} \in\left[0, \mathbf{X}_{\max }\right]$, as $N_{1}$ and $N_{2}$ approach infinity, the aggregate demand for $Y$ converges uniformly to

$$
\mathbf{Y}(\mathbf{X})=\left\{\begin{aligned}
-\frac{y_{1, \max }}{x_{1, \max }} \mathbf{X}+\mathbf{Y}_{\max }, & \mathbf{X} \in\left[0, N_{1} \cdot x_{1, \max }\right] ; \\
-\frac{y_{2, \max }}{x_{2, \max }}\left(\mathbf{X}-N_{1} \cdot x_{1, \max }\right)+N_{2} \cdot y_{2, \max }, & \mathbf{X} \in\left(N_{1} \cdot x_{1, \max }, \mathbf{X}_{\max }\right] .
\end{aligned}\right.
$$

This piecewise linear function is continuous and convex.

Proposition 4 demonstrates the conditions under which an economy of individual risk seekers aggregates to an economy with strictly convex, but not differentiable, indifference curves. We can take the analysis one step further by demonstrating conditions under which the aggregate indifference curves are differentiable. This requires a large number of wealth classes associated with a large number of marginal rates of substitutions. As in the case with two wealth classes, the aggregate indifference curves
Figure 3 Aggregate Indifference Curve with Four Agent Types

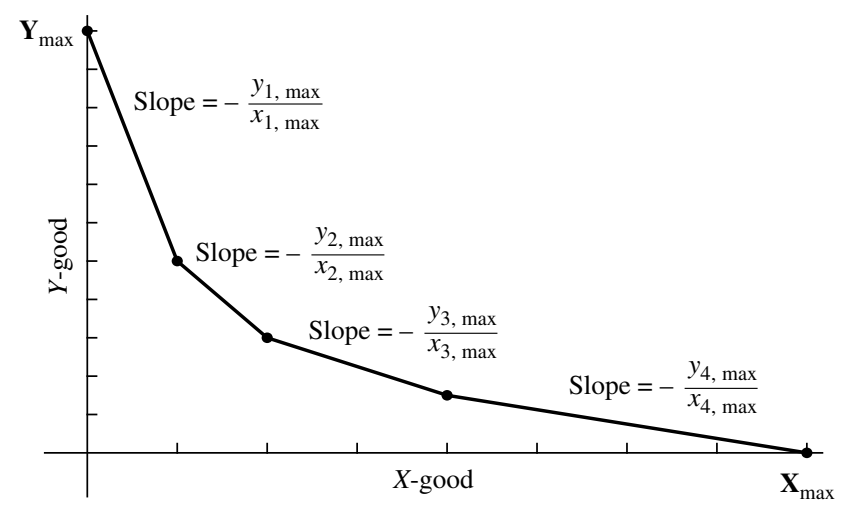

Notes. We plot the aggregate indifference curve in the limit as the number of risk-seeking agents in each of the four wealth classes increases to infinity. Agents fall into four groups such that $\left|y_{i, \max } / x_{i, \max }\right|>\left|y_{i+1, \max } / x_{i+1, \max }\right|$ for $i=1,2,3$. The aggregate indifference curve is piecewise linear and convex.

are piecewise linear but with many linear segments. With an infinite number of wealth classes (heterogeneous distribution of wealth), each linear segment is infinitely short, and the change in the rate between linear segments is infinitely small. This gives rise to a continuous and differentiable curve in the limit.

Assume there are $w$ wealth classes. Each wealth class $i$ has a sufficiently large number of agents, $N_{i}$, so that the indifference curve is arbitrarily close to a straight line, as in Proposition 4 . The agents are ordered by their indifference curves, $k_{i}<k_{i+1}$, so that $\left|y_{i, \max } / x_{i, \max }\right|>\left|y_{i+1, \max } / x_{i+1, \max }\right|$. Following the same argument as presented in Proposition 4, for wealth classes $i=1$ to $w$, the piecewise linear aggregate demand for $Y$ is given by the system:

$\mathbf{Y}_{i}(\mathbf{X})=-\frac{y_{i, \max }}{x_{i, \max }}\left[\mathbf{X}-\mathbf{X}_{i-1}\right]+\mathbf{Y}_{i-1}$ for $\mathbf{X} \in\left[\mathbf{X}_{i-1}, \mathbf{X}_{i}\right]$,

where

$$
\begin{gathered}
\mathbf{X}_{i}=\sum_{j=1}^{i} N_{j} x_{j, \max }, \quad \mathbf{Y}_{i}=Y_{\max }-\sum_{j=1}^{i} N_{j} y_{j, \max }, \\
\mathbf{X}_{0}=0, \quad \mathbf{Y}_{0}=Y_{\max } .
\end{gathered}
$$

Figure 3 illustrates this piecewise linear function for $w=4$. The function is continuous and convex, but not differentiable. The following theorem shows the existence of a wealth distribution such that the resulting aggregate indifference curve is convex and differentiable.

THEOREM 1. Assume an economy of risk-seeking agents with the same utility function but different levels of wealth. Let there exist $\left[k_{\min }, k_{\max }\right]$, a continuum of utility levels with $k_{\min }>0$ and $k_{\max }<\infty$, and for each level of utility $k_{i} \in\left[k_{\min }, k_{\max }\right]$ there exists a sufficiently large number of agents so that the ith type of agent has a 
demand function described by (2). If for all $k_{i}<k_{i+1}$, $\left|y_{i, \max } / x_{i, \max }\right|>\left|y_{i+1, \max } / x_{i+1, \max }\right|$ and fixing the maximum quantity of $X$ and $Y$ at $\mathbf{X}_{\max }$ and $\mathbf{Y}_{\max }$, respectively, then the aggregate indifference curve for the economy is strictly convex and differentiable over $\left[0, \mathbf{X}_{\max }\right]$. If for all $k_{i}<k_{i+1},\left|y_{i, \max } / x_{i, \max }\right|=\left|y_{i+1, \max } / x_{i+1, \max }\right|$, then the aggregate indifference curve is convex (linear).

Theorem 1 states that the aggregate indifference curve has the same shape as the indifference curve produced by a concave (risk-averse) utility function. Though each individual agent is risk seeking, the economy behaves as if it is risk averse!

We have shown that an economy of risk seekers, each facing a budget constraint, leads to aggregate preferences that are not risk seeking. When all agents are identical, the aggregate preferences are consistent with risk neutrality. When we relax the homogeneity assumption to allow for a continuum of wealth classes, the aggregate economy is risk averse with a smooth indifference curve. Perfect competition, budget constraints, and heterogeneity change the appearance of risk-seeking individuals to a riskaverse aggregate market.

\section{Disaggregating the Economy}

An economy consisting of risk-seeking agents can lead to an aggregate economy that is risk averse. We take this analysis one step further by performing the previous analysis backward. We start with an aggregate convex indifference curve, implying that the economy is risk averse. We then prove the existence of risk-seeking agents that aggregate to the same risk-averse aggregate indifference curve. Any economy exhibiting aggregate risk-averse behavior can be supported by risk-seeking individuals. The result obtains because all the steps in the analysis in the previous section can be performed in the reverse order. ${ }^{10}$

THEOREM 2. Consider an economy of risk-averse agents with an aggregate indifference curve defined by $\mathbf{Y}=g(\mathbf{X})$ with the properties $-\infty<g^{\prime}(\mathbf{X})<0, g^{\prime \prime}(\mathbf{X})>0$ for $\mathbf{X} \in$ $\left[0, \mathbf{X}_{\max }\right], g(0)=\mathbf{Y}_{\max }$ and $g\left(\mathbf{X}_{\max }\right)=0$. There exists a distribution of wealth such that an economy of individual riskseeking agents with indifference curves $y(x)=\Psi\left(x ; k_{i}\right)$ satisfying the property $\left|y_{i, \max } / x_{i, \max }\right|>\left|y_{i+1, \max } / x_{i+1, \max }\right|$ for $k_{i}<k_{i+1}$ has the same aggregate demand (aggregate indifference curve) as the economy of risk-averse agents.

This is an important result. Aggregate behavior does not imply the same individual behavior. Specifically, a risk-averse economy does not imply riskaverse individuals.

${ }^{10}$ Others who have considered the question of disaggregation are Chiappori and Ekeland (1999), Gottardi and Hens (1999), and Geanakoplos and Polemarchakis (1980).

\section{Discussion}

The primary implications of our results are for the aggregation of nonstandard preferences. ${ }^{11}$ Though the literature describing the behavioral biases and nonstandard preferences of individuals is large, we are unaware of any paper specifically addressing the aggregation of such preferences in any setting. We show that the relation between individual behavior and aggregate market behavior is not obvious and can be very complex to derive. In this section, we discuss our results in the context of the literature and highlight empirical and theoretical implications of our results.

\subsection{Agent Preferences and Market Frictions}

The behavioral literature has made an effort to describe observed individual behavior in a utility framework. For example, Kahneman and Tversky (1979) observed that individuals underweight uncertain outcomes and overweight certain outcomes. As a result, individuals are risk averse over gains and risk seeking over losses. Friedman and Savage (1948) and, subsequently, Markowitz (1952) observed that individuals buy both lottery tickets and insurance. They argued that agents must be risk seeking over a range of gains and risk averse over a range of losses. It is interesting to note that these utility functions were hypothesized to explain the behavior of individuals and not to explain economic aggregates.

There are many utility functions that attempt to describe various observed patterns of behavior, but the modeling of market frictions (consumption restrictions, present and future expected interest rates, investment opportunities, tax structure, borrowing restrictions, transaction costs, and so on) is as important as the choice of the utility function (Hakansson 1970). The underlying preferences of individuals may be modified significantly in the presence of such frictions. To illustrate this point, Hakansson (1970) modeled a risk-averse agent facing a nonlinear borrowing constraint. The derived utility of such an investor is consistent with the Friedman-Savage utility function, even though the agent is risk averse at each point in time. ${ }^{12}$ We add to this literature by showing

${ }^{11}$ For a survey of the aggregation literature, see Blundell and Stoker (2005). See Eisenberg (1961) for aggregation of concave utility functions.

${ }^{12}$ Several other studies have shown market environment and frictions can impact agents' preferences. Appelbaum and Katz (1981) presented a model similar to Hakansson (1970). Risk-averse agents can appear to display risk-seeking behavior if they face the possibility of bankruptcy (Lippman et al. 1980), if they face nonlinear budget constraints (Bordley 1995), or if by taking a gamble the agent can acquire important information (Baron 1974, McCardle and Winkler 1992). Postlewaite et al. (2008) modeled risk-neutral agents facing consumption commitments. Such agents exhibit riskaverse behavior over small variations in income. 
that the existence of a budget constraint can distort preferences in the aggregate. Individuals may have risk-seeking utility functions even if the aggregate economy appears risk averse. This is an important result because aggregate prices are determined by aggregate preferences.

Moreover, constraints can encourage rationality in aggregate markets. Becker (1962) showed that individuals who irrationally choose consumption will still have a downward-sloping demand curve. Such individuals are subject to changing opportunity sets that force the agents to make choices that appear rational. Becker (1962, p. 8) concluded, "Households may be irrational and yet markets quite rational." In an interesting experiment by Gode and Sunder (1993), "zerointelligent" computers with no profit motives submit completely random limit orders. The zero-intelligent computers face a constraint that prevents them from trading at a loss. Despite the randomness of trading, the zero-intelligent computers are able to arrive at the correct equilibrium price. When the constraint is removed, prices fail to converge to their equilibrium value. The existence of market "rules" causes random traders to appear rational. In a follow-up paper, Gode and Sunder (1997) theoretically showed the importance of market rules in causing allocative efficiency. Budget constraints, when binding, prevent the observed portfolios from reflecting the economic agents' true underlying preferences. We show that risk seekers facing perfect competition and a budget constraint appear to be risk averse in the aggregate.

The psychology literature has already provided fruitful direction for asset pricing by describing the behavioral biases of individuals. With respect to theory, to use this information to explain economic aggregates we need to build models with rich market environments (Hakansson 1970), understanding that the market environment can distort the aggregate demands of individuals with behavioral biases. It is not at all obvious what aggregate demands look like. In addition to modeling a rich market environment, it is necessary to explicitly aggregate agents' preferences. We show that aggregate preferences do not necessarily reflect risk-seeking individual behavior because of the constraints and competitiveness contained within the overall economy.

The empirical research has tended to lie in two separate camps. The first line is the research studying investor preferences by interpreting the choices of individuals. The second line involves measuring market aggregates. An interesting direction for empirical research is to bridge the two lines by devising experiments similar to that of Gode and Sunder (1993). In such an experiment, the researcher is able to observe the behavior of each individual, control the constraints within the experimental economy, and observe the movement of the aggregate prices. The entire process, beginning with heterogeneous individuals and ending with aggregate prices, can be observed. In such a setting, various constraints can be imposed to observe their effect on the aggregate market. ${ }^{13}$

Controlled experiments can also help gain understanding of the impact of heterogeneity on the equilibrium risk premium. In our market of risk seekers, greater heterogeneity is related to differences in the trade-off between the sources of utility. Large heterogeneity creates a large curvature in the aggregate indifference curve, leading to a large risk premium. Alternatively, in a market of risk-averse investors with negative exponential utility functions (for example), heterogeneity in wealth is directly linked to aggregate risk aversion, with wealthier agents having a larger direct impact on the aggregate (the risk tolerance of each agent is weighted by the agent's wealth). Fluctuations in the distribution of wealth directly affect aggregate risk aversion and the risk premium in predictable ways. Controlled experiments can provide an interesting setting in which to investigate the connections between heterogeneity and prices. Whereas many experiments tend to use a group of university students (a fairly homogeneous collection of individuals), the careful construction of a group of test subjects that focuses on a particular definition of heterogeneity (age, wealth, gender, etc.) will lead to a better understanding of how heterogeneity affects aggregate pricing. ${ }^{14}$

In addition to controlled experiments, there are many natural experiments that one can consider. Market constraints (e.g., taxes, trading costs, and no short selling) can change over time. If these constraints affect the trading behavior of a sufficiently large collection of investors, a change in the constraint may be followed by observable changes in individual demands and aggregate prices. When new regulations are imposed, what happens to the risk premium, the portfolios held by investors, and the revealed risk

\footnotetext{
${ }^{13}$ See Bossaerts (2002) for a description of other types of experiments that can be extended to incorporate constraints.

${ }^{14}$ To test the effects of heterogeneity on risk premium in the data, one must compare two (or more) markets with different levels of heterogeneity. One approach may involve investigating risk premium through time and relating it to the heterogeneity of investors. Although we suggest that conducting precise empirical tests is outside of the scope of this paper, one may envision a test, for example, based on the notion that older people have greater risk aversion than young people. One may then attempt to measure the degree of heterogeneity among investors by looking at periods when there are more older (younger) investors in the market. The Consumer Expenditure Survey data set can be used to determine the individual characteristics of people receiving income from dividends and interest. This can potentially give an idea of the heterogeneity in the market.
} 
preferences? For example, if a no-short-sale constraint makes it harder to implement hedging, does it result in (1) investors holding riskier positions, giving the appearance of low risk aversion, or (2) investors not trading in the asset that cannot be hedged, giving the appearance of high risk aversion? Such a change in regulation occurred in September 2008 when the Securities and Exchange Commission banned short selling in response to the sudden market crash. ${ }^{15}$

\subsection{The Individual and the Aggregate}

Representative agent asset-pricing models traditionally begin with a set of risk-averse individuals and end with a set of (testable) Euler equations describing the moments of economic aggregates. The literature has advanced in two ways. On one hand, spurred by the empirical failure of traditional models to explain economic aggregates, variations have been suggested to improve their empirical predictions. On the other hand, spurred by the inadequate modeling of observed individual behavior, new utility functions have been introduced. Without studying the aggregate properties of the newly introduced utility functions, their effect on economic aggregates is unknown.

Although many generalizations of traditional models have been suggested, two are particularly relevant. Heaton and Lucas (1992) studied the impact of market frictions (borrowing constraints, short-sale constraints, and borrowing costs) on asset prices in an economy where income shocks are uninsurable; Constantinides and Duffie (1996) considered a similar economy but with heterogeneous consumers (in terms of labor income) and incomplete consumption insurance. ${ }^{16}$ The generalizations made in both models aid in matching economic aggregates, but more related to this paper, market frictions and heterogeneity drive a wedge between the individual and the aggregate. Uninsurable income shocks lead to high volatility in individual consumption but low volatility in aggregate consumption. Constantinides and Duffie (1996) found that although all individuals have identical levels of risk aversion, the empirical researcher who ignores heterogeneity will estimate a different level of risk aversion for the representative agent. ${ }^{17}$ As in the analysis of the risk-seeking agents in this paper, Constantinides and Duffie (1996) found that the assumptions of income shocks and incomplete

\footnotetext{
${ }^{15}$ In addition, studies such as Goetzmann and Massa (2002) can take advantage of trading data at the level of individual investors to work on establishing connections between individual trading activity and aggregate prices.

${ }^{16}$ See Heaton and Lucas (1992), Constantinides and Duffie (1996), and references therein for more discussion of deviations from the canonical representative agent model.

${ }^{17}$ Risk aversion is overestimated when the cross-sectional distribution of consumption growth increases during economic bad times.
}

consumption insurance lead to a no-trade equilibrium where agents disagree on the marginal rate of substitution.

Even larger deviations from the traditional paradigm have been proposed in the behavioral literature by arguing that risk aversion alone is not sufficient for describing consumer decisions (Shefrin 2005). Jarrow (1988) showed that the arbitrage pricing theory holds even when agents do not have preferences that can be represented by risk-averse expected utility functions. Investors with nonstandard utility functions hold different portfolios than risk-averse agents do (Jarrow and Zhao 2006, Bawa 1977). Shefrin and Statman (2000) developed a behavioral portfolio theory, where investors chose portfolios by considering expected wealth, desire for security and potential, aspiration levels, and probabilities of achieving aspiration levels. The optimal portfolios of behavioral portfolio theory investors resemble combinations of bonds and lottery tickets. Bakshi and Chen (1996) studied, both theoretically and empirically, an economy where investors acquire wealth not only for its implied consumption but also for the resulting social status. When investors care about relative social status, their social standards affect their consumption and their risk-taking behavior, thus impacting asset prices. Levy and Levy (2002) found strong empirical support for the Markowitz utility function in an experimental study. ${ }^{18}$

The behavioral literature has made important strides in describing individual preferences, but it is aggregate behavior that determines prices, aggregate consumption, volatility, and other economic aggregates. We show in this paper that aggregation-the bridge connecting individual preferences and economic aggregates-is an important and necessary next step in the evolution of this literature. It cannot be immediately assumed that the effects of individual biases should appear in aggregate data. ${ }^{19}$

\footnotetext{
${ }^{18}$ There has also been much evidence of risk-seeking behavior. Goetzmann and Massa (2002) identified a group of index fund investors who systematically invest in the fund after volatility increases. Coval and Shumway (2005) found evidence that proprietary traders are loss averse (risk averse over gains and risk seeking over losses). Many others have found evidence of riskseeking behavior in prices (Jackwerth 2000; Levy and Levy 2001, 2002; Rosenberg and Engle 2002; Kliger and Levy 2002; Bakshi and Wu 2010; Green and Rydqvist 1997; Florentsen and Rydqvist 2002), and the evidence spans time, several countries, and many different empirical methods.

${ }^{19}$ We refer to Kirman (1992) for a related general discussion. He argued that reduction of the behavior of a group of agents to one representative agent is "not simply an analytical convenience as often explained, but is both unjustified and leads to conclusions which are usually misleading and often wrong" (p. 117). Kirman (1992) suggested that it is essential to study aggregates that emerge when agents interact with each other. Our analysis is consistent with these arguments.
} 
Our results suggest that nonstandard utility functions may lead to new interpretations of empirical findings. We show that an economy demanding a risk premium can be formed from individuals who do not demand such compensation, and individuals who do not demand compensation for risk can lead to an economy that does demand a risk premium. These results are staggering when contrasted with a more conventional intuition.

Consider a well-known case. Suppose we assume that the representative agent of the economy has, for example, a negative exponential utility function. By observing returns on the market (an aggregate quantity), we infer that the risk aversion of the aggregate representative investor must equal some value, $R A_{A}$. If we now follow the arguments that lead to the equity premium puzzle, judgment is applied to the value of $R A_{A}$, leading us to conclude that the representative agent's risk-aversion level is too high. But how does one judge whether risk aversion of an aggregate agent-an abstract construct by definition-is too high or too low? In the case of individuals, the level of risk aversion has implications for behavior, thus enabling us to make judgments about high and low levels of aversion to risk. In the aggregate case, such judgment is harder to make. One therefore is tempted to interpret the equity premium puzzle by taking the additional step of placing judgment on the individuals who make up the economy. ${ }^{20}$

To explore this in more detail, consider the negative exponential utility function. It is well known that when all individuals have negative exponential utility, the representative investor also has negative exponential utility with risk tolerance (the inverse of risk aversion) equal to the wealth-weighted harmonic mean of the individual agents' risk tolerance:

$$
\frac{1}{R A_{A}}=\sum_{k=1}^{K} \frac{W_{k}}{W_{A}} \frac{1}{R A_{k}}, \quad W_{A}=\sum_{k=1}^{K} W_{k} .
$$

Harmonic means are never larger than arithmetic means and are equal only when all individuals are equally risk averse. Therefore, the risk aversion of

\footnotetext{
${ }^{20}$ Although it may be tempting to make statements concerning individual behavior based on aggregate data, our results suggest that this may lead to wrong conclusions. In an overview article, Kirman (1992) pointed out that in empirical tests based on the representative agent paradigm, there is a fundamental joint hypothesis problem: "If one rejects a particular behavioral hypothesis, it is not clear whether one is really rejecting the hypothesis in question, or rejecting the additional hypothesis that there is only one individual" (p. 118). Kirman (1992, p. 118) also pointed out that "The sum of the behavior of simple economically plausible individuals may generate complicated dynamics, whereas constructing one individual whose behavior has these dynamics may lead to that individual having very unnatural characteristics." Our formal results are consistent with these observations.
}

the aggregate representative investor is less than the wealth-weighted arithmetic average of the individuals' risk aversion. If the representative agent's risk aversion is already deemed too high, then there will be individuals with even more implausibly high levels of risk aversion. In this conventional framework, the finding of a high-risk premium in the aggregate first leads to an implausible level of aggregate risk aversion, which then leads to an even more implausible conclusion about the individual agents. The conclusion that individual agents have implausibly high levels of risk aversion is obtained in this setting because of the jump from a risk-averse representative agent with exponential utility to risk-averse individuals with exponential utility. In sum, if one builds the aggregate economy by assuming that the individuals who make up the economy have negative exponential utility, the puzzle is only further deepened.

In contrast, consider our disaggregation result. ${ }^{21}$ Observing that the risk aversion of the representative agent is too large is only problematic if it implies unreasonable individual behavior. The puzzle is diminished if it is possible to have a large risk aversion of the representative agent simultaneously with a more reasonable assumption on the preferences of individuals. We show that such a possibility exists. A risk-averse representative agent may be found by aggregating risk-averse individuals; but it can also be obtained by aggregating risk-seeking individuals. Utility functions that include risk-seeking regions may not exacerbate the risk premium puzzle. These functions may actually lead to more reasonable conclusions regarding the individual agents who support the economy. Our analysis relies on the notion that when we observe a person's choices (portfolio), we observe not just their utility function but also all of the economic constraints and competition they face within the economy.

\section{Conclusion}

The relation between risk preferences of individual agents in the economy and the attitude toward risk in the aggregate is fundamental in financial economics. Ultimately, aggregate preferences determine the price of risk in the economy. Yet individual biases characterize deviations from canonical fully rational financial models. In this paper, we establish new and important results about the link between individual and aggregate preferences. We show that there can be significant differences in the nature of risk preferences of individual investors and aggregate preferences toward risk in the economy.

\footnotetext{
${ }^{21}$ In a different setting, Hellwig $(1980,1982)$ studied an economy in which all agents have a negative exponential utility. The focus of his study, however, is on information, and not on the level of expected returns.
} 
We consider an economy where all individual agents are risk seeking, face a budget constraint, and operate under perfect competition. We prove several theorems, working "up" from individual agents to the aggregate economy. Our main result in this section shows that an economy that consists of small (atomistic) risk-seeking individuals in the aggregate can be characterized by an indifference curve consistent with risk aversion. This result emphasizes the importance of aggregating individual demands when building models. Aggregate preferences can be vastly different from the preferences of the individuals supporting the economy.

Our last result is perhaps even more important. We start with an aggregate convex indifference curve corresponding to the case of a risk-averse aggregate economy and work "down" to the individual agents. We show that there exists an economy composed of risk-seeking individuals and a distribution of wealth such that the aggregate economy produces the same risk-averse indifference curve as given. A risk-averse economy need not imply riskaverse individuals. From a theoretical standpoint, this result is important because it increases our understanding of the link between aggregate models and individuals. From an empirical standpoint, studies based on aggregate data can potentially be consistent with a wide variety of individual investor behavior specifications-even the ones based on utility functions with convex regions. Therefore, caution must be taken when drawing conclusions about individual behavior based on aggregate data.

We are certain that models can be constructed such that the aggregation of nonstandard individual preferences results in nonstandard aggregate preferences. Such examples further illustrate the necessity of aggregation. Market frictions, agent heterogeneity, and perfect competition have nontrivial effects when bridging the gap between individuals and the aggregate market. Our results suggest that understanding the aggregate properties of economies populated by agents with nonstandard utilities is an important area for continued research.

\section{Acknowledgments}

The authors are grateful to departmental editor Wei Xiong, previous departmental editor David Hsieh, an anonymous associate editor, and three anonymous referees for insightful and constructive comments that have greatly improved the paper. The authors also thank Peter Bossaerts, John Cochrane, Ned Elton, Michael Gallmeyer, John Geanakoplos, William Goetzmann, Craig Holden, Jonathan E. Ingersoll Jr., Ravi Jagannathan, Robert Jennings, Peng Liu, Stavros Panageas (discussant), Thierry Post, Jun Qian, Robert Savickas, Charles Trzcinka, Gregory Udell, and Fwu-Ranq Chang, as well as participants of the Frontiers of Finance 2005 (Bonaire), 2006 FMA Meeting (Salt Lake
City), Washington Area Finance Association 2005, and the Risk Theory Society Meeting 2007 conferences for helpful comments and suggestions. A part of this research was conducted when Andrey Ukhov was at Kellogg School of Management, Northwestern University.

\section{Appendix}

\section{Homogeneous Risk-Seeking Agents}

Proof of Proposition 1 (Efficient Allocation for IdenTiCAL Agents). First notice that each agent's indifference curve, $y=\Psi(x ; k)$, is concave and continuous for all $x$ because, by the implicit function theorem,

$$
\frac{d^{2} y}{d x^{2}}=-\frac{u_{x x} u_{y}^{2}-2 u_{x y} u_{x} u_{y}+u_{y y} u_{x}^{2}}{u_{y}^{3}}<0
$$

Note that $u_{x y}=0$ because we study an additively separable utility function. Because the sum of concave functions is concave, the interior optimum is a maximum and not a minimum. Because of the nonnegativity constraints, the set of feasible solutions is compact; therefore, the solution exists and the solution must be located on the boundary of the feasible solution set.

We use proof by contradiction. Suppose there exists an efficient allocation such that two or more agents hold positive quantities of both $X$ and $Y$. Without loss of generality, assume that agent $i$ holds the allocation $\left(x_{i}, y_{i}\right)$ and agent $j$ holds $\left(x_{j}, y_{j}\right)$ such that $x_{i} \geq x_{j}>0$. Let $\epsilon>0$ be an arbitrarily small quantity of $X$ and let $s=\left(y_{j}-y_{i}\right) /\left(x_{i}-x_{j}\right)$ (or $s=-\partial y / \partial x$ if $x_{i}=x_{j}$ ). Consider the possible trade between agents $i$ and $j$. Agent $j$ gives $\epsilon$ of the $X$ good in exchange for $s \epsilon$ of the $Y$ good. Agent $i$ 's new allocation is $\left(x_{i}+\epsilon\right.$, $\left.y_{i}-s \epsilon\right)$ and agent $j$ 's new allocation is $\left(x_{j}-\epsilon, y_{j}+s \epsilon\right)$. However, by the concavity of the indifference curve, $y\left(x_{i}\right)-$ $s \epsilon>y\left(x_{i}+\epsilon\right)=\Psi\left(x_{i}+\epsilon ; k\right)$ and $y\left(x_{j}\right)+s \epsilon>y\left(x_{j}-\epsilon\right)=$ $\Psi\left(x_{j}-\epsilon ; k\right)$. Through trade, both agents improve their utility contradicting the claim that the allocation was efficient. Hence, at most one agent will hold a combination of $X$ and $Y$. This implies that a set of agents will hold only $X$ so that the market for $X$ clears and in equal quantities because of homogeneity. The remaining agents must hold only $Y$ in equal quantities, such that each agent has utility $k$.

Proof of Proposition 2. To model the increasing size of the economy, we start with $N>0$ agents and then take the limit as $N$ approaches infinity. So that the economies in the sequence are comparable, we fix $\mathbf{X}_{\max }$ and $\mathbf{Y}_{\max }$. Therefore, $x_{\max , N}=\Psi^{-1}\left(0 ; k_{N}\right)=\mathbf{X}_{\max } / N$ and $y_{\max , N}=\Psi\left(0 ; k_{N}\right)=$ $\mathbf{Y}_{\max } / N$ both tend toward zero as the number of agents increases. We include the subscript $N$ to highlight dependence on the number of agents.

For some integer $n=0,1,2,3, \ldots, N$, we write $\mathbf{X} \in$ $\left[0, \mathbf{X}_{\max }\right]$ as $\mathbf{X}=n x_{\max , N}+r_{N}$ for $r_{N} \in\left[0, x_{\max , N}\right)$. By Proposition $1, n$ agents hold only the $X$ good, one agent holds the allocation $(r, y(r))$, where $y(r)=\Psi(r, k)$, and $N-n$ agents hold only the $Y$ good. We write the demand for the $Y$ good as

$$
\mathbf{Y}_{N}(\mathbf{X})=(N-n) y_{\max , N}+y\left(r_{N}\right) .
$$

To show that $\mathbf{Y}_{N}(\mathbf{X})$ approaches $\mathbf{Y}(\mathbf{X})$ uniformly, we must show that for any $\epsilon>0$ there exists an $N^{*}>0$ such that for 
all $N \geq N^{*},\left|\mathbf{Y}_{N}(\mathbf{X})-\mathbf{Y}(\mathbf{X})\right|<\epsilon$. Let $\epsilon>0$ and define $N^{*}=$ $2 \mathrm{Y}_{\max } / \epsilon$. Hence, for $N>N^{*}$

$$
\begin{aligned}
& \left|\mathbf{Y}_{N}(\mathbf{X})-\mathbf{Y}(\mathbf{X})\right| \\
& \quad=\left|(N-n) y_{\max , N}+y\left(r_{N}\right)-\left(-\frac{y_{\max , N}}{x_{\max , N}} \mathbf{X}+\mathbf{Y}_{\max }\right)\right| \\
& \quad=\left|-n y_{\max , N}+y\left(r_{N}\right)+\frac{y_{\max , N}}{x_{\max , N}} \mathbf{X}\right| \\
& \quad=\left|-\frac{\mathbf{X}-r_{N}}{x_{\max , N}} y_{\max , N}+y\left(r_{N}\right)+\frac{y_{\max , N}}{x_{\max , N}} \mathbf{X}\right| \\
& \quad=\left|\frac{r_{N}}{x_{\max , N}} y_{\max , N}+y\left(r_{N}\right)\right| \leq\left|\frac{r_{N}}{x_{\max , N}} y_{\max , N}\right|+\left|y\left(r_{N}\right)\right| \\
& \quad \leq 2 y_{\max , N} \leq 2 y_{\max , N^{*}}=\epsilon .
\end{aligned}
$$

The second equality comes from $\mathbf{Y}_{\max }=N y_{\max , N}$, and the third equality uses $\mathbf{X}=n x_{\max , N}+r_{N}$. The triangle inequality is used to show the first inequality, the second inequality uses the observation that $r_{N} / x_{\max , N}<1$ and $y\left(r_{N}\right) \leq y_{\max }$, and the last inequality is true because $y_{\max }$ is a decreasing function of $N$ and $N>N^{*}$.

\section{Heterogeneous Risk-Seeking Agents}

Proof of Proposition 3. It has been established in Proposition 1 that when agents are risk seeking, then at most one agent can hold positive quantities of both the $X$ and $Y$ goods. It remains to be shown that when $\mathbf{X}<N_{1} x_{1 \text {, max }}$ it is efficient to allocate all the $X$ good to the type 1 agents. The type 2 agents only hold $X$ when $\mathbf{X}>N_{1} x_{1, \max }$.

Suppose the economy is initially endowed with $\mathbf{Y}_{\max }$ and none of the $X$ good. The $Y$ good is distributed in such a way that all type 1 agents receive the allocation $\left(0, y_{1, \max }\right)$ and all type 2 agents receive $\left(0, y_{2, \max }\right)$. Now suppose $X=\epsilon \leq$ $x_{1, \max } \leq x_{2, \max }$ is available. For $\epsilon$ to be allocated to a type 1 agent, it is sufficient to show that $\left|y_{1}^{\prime}(0)\right|>\left|y_{2}^{\prime}(0)\right|$, indicating that the type 1 agents are willing to substitute more $Y$ for the initial unit of $X$ received.

Because the utility function is additively separable, $u_{x}(\cdot)$ is a function of $x$ only, and $u_{y}(\cdot)$ is a function of $y$ only. Both partial derivatives are positive because $u(x, y)$ is an increasing function in both $x$ and $y$. By the implicit function theorem,

$$
\left.\frac{d y_{1}}{d x}\right|_{x=0, k=k_{1}}=-\frac{u_{x}(x)}{u_{y}(y)} \text { and }\left.\frac{d y_{2}}{d x}\right|_{x=0, k=k_{2}}=-\frac{u_{x}(x)}{u_{y}(y)} .
$$

Because the derivative is evaluated at $x=0$ and agents have the same utility function differing only in wealth, it must be that the only difference is in the allocation of $y$. Then the numerators are equal because they are a function of $x$ only. By convexity of $u(x, y)$, the partial derivative $u_{y}(y)$ is higher for higher values of $y$. Then the denominator is larger in the second ratio and therefore

$$
\left|y_{1}^{\prime}(0)\right|=\left.\left|\frac{d y_{1}}{d x}\right|_{x=0, k=k_{1}}|>| y_{2}^{\prime}(0)|=| \frac{d y_{2}}{d x}\right|_{x=0, k=k_{2}} \mid .
$$

The inequality $\left|y_{1}^{\prime}(0)\right|>\left|y_{2}^{\prime}(0)\right|$ holds for an arbitrary $u(x, y)$ that satisfies our conditions of differentiability, monotonicity, and convexity. Hence, it is efficient to allocate $\epsilon$ to a single type 1 agent.
At most one agent can hold an interior position and the rate of substitution increases (in absolute value) with each additional unit of $X$, so any additional $X$ must be allocated to the single agent holding the interior allocation until the agent holds $\left(x_{1, \max }, 0\right)$. The argument repeats itself with $N_{1}+N_{2}-1$ agents holding all $Y$ and one agent holding only $X$. Hence, by the concavity of the indifference curve, the $X$ consumption good will be allocated to the type 1 agents until all agents receive $x_{1, \max }$ or the supply of $X$ has been depleted. If all type 1 agents have $x_{1 \text { max }}$, then the remaining supply of $X$ will be allocated to the type 2 agents one agent at a time, with each type 2 agent holding no more than $x_{2, \max }$.

Proof of Proposition 4. The proof is similar to the proof of Proposition 2. Define $\mathbf{X}_{1, \max }=N_{1} x_{1, \max }, \mathbf{X}_{2, \max }=$ $N_{2} x_{2, \max }, \mathbf{Y}_{1, \max }=N_{1} y_{1, \max }$, and $\mathbf{Y}_{2, \max }=N_{2} y_{2, \max }$. As before, we hold constant $\mathbf{X}_{\text {max }}=\mathbf{X}_{1, \max }+\mathbf{X}_{2, \max }$ and $\mathbf{Y}_{\text {max }}=\mathbf{Y}_{1, \max }+$ $\mathbf{Y}_{2, \max }$ so that the primary difference between the elements of the sequence of economies as $N_{1}$ and $N_{2}$ increase to infinity is the number of agents. Note that this implies that $\mathbf{X}_{1, \max }, \mathbf{X}_{2, \max }, \mathbf{Y}_{1, \max }$, and $\mathbf{Y}_{2, \max }$ are also fixed because as we allow only $N_{1}$ (or alternatively $N_{2}$ ) to increase, $\mathbf{X}_{\max }-$ $\mathbf{X}_{2 \text { max }}$ and $\mathbf{Y}_{\max }-\mathbf{Y}_{2 \text { max }}$ remain fixed.

When $\mathbf{X} \leq \mathbf{X}_{1, \max }$ then by Proposition 3, all type 2 agents hold only the $Y$ good and some number of type 1 agents hold all the $X$ good. Therefore, we can write $\mathbf{X}=n x_{1, \max }+r_{1}$ for $r_{1} \in\left[0, x_{1, \max }\right)$ and some integer $n=0,1,2, \ldots, N_{1}$, indicating that $n$ type 1 agents hold only the $X$ good and one agent holds an interior allocation. The aggregate demand for the $Y$ good is

$$
\mathbf{Y}_{N}(\mathbf{X})=\mathbf{Y}_{2, \max }+\left(N_{1}-n\right) y_{1, \max }+y\left(r_{1}\right) .
$$

The problem is identical to the case presented in Proposition 2. As $N_{1}$ approaches infinity, $\mathbf{Y}_{N}(\mathbf{X})$ converges to

$$
\mathbf{Y}(\mathbf{X})=\mathbf{Y}_{2, \max }-\frac{y_{1, \max }}{x_{1, \max }} \mathbf{X}+\mathbf{Y}_{1, \max }=-\frac{y_{1, \max }}{x_{1, \max }} \mathbf{X}+\mathbf{Y}_{\max }
$$

uniformly on $\mathbf{X} \in\left[0, \mathbf{X}_{1, \max }\right]$.

When $\mathbf{X}>\mathbf{X}_{1 \text { max }}$, by Proposition 3, all type 1 agents hold only the $X$ good. Therefore, we can write $\mathbf{X}=\mathbf{X}_{1 \text {, max }}+$ $n x_{2, \max }+r_{2}$, which can be rewritten as $\mathbf{X}^{*}=\mathbf{X}-\mathbf{X}_{1, \max }=$ $n x_{2, \max }+r_{2}$ for $r_{2} \in\left[0, x_{2, \max }\right)$ and some integer $n=$ $0,1,2, \ldots, N_{2}$. The aggregate demand for the $Y$ good is

$$
\mathbf{Y}_{N}(\mathbf{X})=\left(N_{2}-n\right) y_{2, \max }+y\left(r_{2}\right)
$$

The problem is again identical to the case presented in Proposition 2. As $N_{2}$ approaches infinity, $\mathbf{Y}_{N}(\mathbf{X})$ converges uniformly to

$$
\begin{aligned}
\mathbf{Y}(\mathbf{X}) & =-\frac{y_{2, \max }}{x_{2, \max }} \mathbf{X}^{*}+N_{2} \cdot y_{2, \max } \\
& =-\frac{y_{2, \max }}{x_{2, \max }}\left(\mathbf{X}-\mathbf{X}_{1, \max }\right)+N_{2} \cdot y_{2, \max }
\end{aligned}
$$

for $\mathbf{X} \in\left(X_{1, \max }, \mathbf{X}_{\max }\right]$.

The aggregate indifference curve is clearly continuous and is convex because $\left|y_{1, \max } / x_{1, \max }\right|>\left|y_{2, \max } / x_{2, \max }\right|$. 
Proof of Theorem 1. From our previous results, we know that for $w$ wealth classes, the indifference curve is characterized by a system of $w$ linear functions

$$
\mathbf{Y}_{i}(\mathbf{X})=-\frac{\Psi\left(0 ; k_{i}\right)}{\Psi^{-1}\left(0 ; k_{i}\right)}\left[\mathbf{X}-\mathbf{X}_{i-1}\right]+\mathbf{Y}_{i-1} \quad \text { for } \mathbf{X} \in\left[\mathbf{X}_{i-1}, \mathbf{X}_{i}\right]
$$

where $\mathbf{X}_{i}=\sum_{j=1}^{i} N_{j} x_{j, \max }, \mathbf{Y}_{i}=Y_{\max }-\sum_{j=1}^{i} N_{j} y_{j, \max }, \mathbf{X}_{0}=0$, and $\mathbf{Y}_{0}=Y_{\max }$. The cases $w=1$ (homogeneity) and $w=2$ (two wealth classes) were considered previously. Here we study the case $w \geq 2$. Each wealth class $i=1$ to $w$ is associated with indifference curve $k_{i}$ such that $k_{i}<k_{i+1}$ for all $i$. Hence, there is a one-to-one correspondence between the distribution of wealth and the distribution of indifference curves. We will show that as $w$ increases to infinity, the system $\mathbf{Y}_{i}(\mathbf{X})$ converges to a convex function differentiable for all $\mathbf{X} \in\left[0, \mathbf{X}_{\max }\right]$.

Convexity is immediate by the assumption

$$
\left|\frac{y_{i, \max }}{x_{i, \max }}\right|>\left|\frac{y_{i+1, \max }}{x_{i+1, \max }}\right| \text {. }
$$

To show differentiability, it suffices to show differentiability at each $\mathbf{X}=\mathbf{X}_{i}$, the intersection of two linear segments, because $\mathbf{Y}_{i}(\mathbf{X})$ is clearly differentiable for $\mathbf{X} \in\left(\mathbf{X}_{i-1}, \mathbf{X}_{i}\right)$. For this, it is sufficient to show that $\mid d \mathbf{Y}_{i+1}\left(\mathbf{X}_{i}^{+}\right) / d \mathbf{X}-$ $d \mathbf{Y}_{i}\left(\mathbf{X}_{i}^{-}\right) / d \mathbf{X} \mid<\epsilon$.

The derivative at $\mathbf{X}=\mathbf{X}_{i}$ from the left is

$$
\frac{d \mathbf{Y}_{i}\left(\mathbf{X}_{i}^{-}\right)}{d \mathbf{X}}=-\frac{y_{i, \max }}{x_{i, \max }},
$$

and the derivative from the right is

$$
\frac{d \mathbf{Y}_{i+1}\left(\mathbf{X}_{i}^{+}\right)}{d \mathbf{X}}=-\frac{y_{i+1, \max }}{x_{i+1, \max }} .
$$

Because $y_{\max }=\Psi(0 ; k)>0$ and $x_{\max }=\Psi^{-1}(0 ; k)>0$ are continuous functions of $k$ in $\left[k_{\min }, k_{\max }\right], \Psi(0 ; k) / \Psi^{-1}(0 ; k)$ is also continuous in $k$ for $k$ in $\left[k_{\min }, k_{\max }\right]$. By the definition of continuity, for any $\epsilon>0$, there exists a $\delta>0$ such that if $\left|k_{i}-k_{j}\right|<\delta$ then $\mid \Psi\left(0 ; k_{i}\right) / \Psi^{-1}\left(0 ; k_{i}\right)-$ $\Psi\left(0 ; k_{j}\right) / \Psi^{-1}\left(0 ; k_{j}\right) \mid<\epsilon$. Define the partition on $\left[k_{\min }, k_{\max }\right]$, $P_{k, w}=\left\{k_{1}=k_{\min }, k_{2}, \ldots, k_{w-1}, k_{w}=k_{\max }\right\}$ such that $k_{h+1}=$ $k_{h}+\left(k_{\max }-k_{\min }\right) /(w-1), h=1, \ldots, w-1$. Define $w^{*}=$ $\left(k_{\max }-k_{\min }\right) / \delta+1$. Hence, for any $\epsilon>0$, there exists a $\delta>0$ such that if the number of wealth classes is $w>w^{*}$, then

$$
\left|k_{i+1}-k_{i}\right|=\frac{k_{\max }-k_{\min }}{w-1}<\frac{k_{\max }-k_{\min }}{w^{*}-1}=\delta .
$$

Therefore, the distribution of wealth classes characterized by $k_{i+1}=k_{i}+\left(k_{\max }-k_{\min }\right) /(w-1)$ leads to

$$
\left|\frac{d \mathbf{Y}_{i}\left(\mathbf{X}_{i}^{+}\right)}{d \mathbf{X}}-\frac{d \mathbf{Y}_{i+1}\left(\mathbf{X}_{i}^{-}\right)}{d \mathbf{X}}\right|=\left|\frac{\Psi\left(0 ; k_{i}\right)}{\Psi^{-1}\left(0 ; k_{i}\right)}-\frac{\Psi\left(0 ; k_{i+1}\right)}{\Psi^{-1}\left(0 ; k_{i+1}\right)}\right|<\epsilon
$$

It is straightforward to show that when

$$
\left|\frac{y_{i, \max }}{x_{i, \max }}\right|=\left|\frac{y_{i+1, \max }}{x_{i+1, \max }}\right|
$$

then the aggregate indifference curves are linear.

\section{Disaggregating the Economy}

Proof of Theorem 2. From Theorem 1, we know that an infinitely large number of risk seekers within the same wealth class aggregate to form a chord. Therefore, it is sufficient to show that there exists a particular collection of wealth classes such that the chords formed from each wealth class, characterized by their indifference curve $k_{i}$, uniformly converge to the function $g(\mathbf{X})$. We show how to start with an aggregate convex indifference curve and construct a wealth distribution in an economy where each individual has a concave indifference curve.

Define an initial partition over $\left[0, \mathbf{X}_{\max }\right]$ as $\mathbf{P}_{1}=\left\{\mathbf{X}_{1,1}=\right.$ $\left.0, \mathbf{X}_{1,2}, \ldots, \mathbf{X}_{1, w}=\mathbf{X}_{\max }\right\}$, where $\mathbf{X}_{1, i}=i\left(\mathbf{X}_{\max } / w\right)$ for wealth classes $i=1$ to $w$. Define the sequence of refinements as

$$
\begin{aligned}
\mathbf{P}_{j}= & \left\{\mathbf{X}_{j, 1}=0, \mathbf{X}_{j, 2}, \ldots, \mathbf{X}_{j, w_{j}}=\mathbf{X}_{\max }\right\} \\
= & \left\{\mathbf{X}_{j-1,1}=0, \mathbf{X}_{j-1,2}, \ldots, \mathbf{X}_{j-1, w_{j-1}}=\mathbf{X}_{\max }\right\} \\
& \cup\left\{\mathbf{M}_{j-1,1}, \mathbf{M}_{j-1,2}, \ldots, \mathbf{M}_{j-1, w_{j-1}-1}\right\},
\end{aligned}
$$

where $\mathbf{M}_{j-1, k}=\left(\mathbf{X}_{j-1, k+1}-\mathbf{X}_{j-1, k}\right) / 2$ and $j>1$. The partition $\mathbf{P}_{j}$ includes all elements of $\mathbf{P}_{j-1}$ plus the midpoints between all elements of $\mathbf{P}_{j-1}$. If partition $\mathbf{P}_{j-1}$ has $w_{j-1}$ wealth classes, then partition $\mathbf{P}_{j}$ has $2 w_{j-1}-1$ wealth classes.

Each wealth class is characterized by the indifference curve of agents in it. For partition $\mathbf{P}_{j}$, choose $\mathbf{Y}_{j, i}\left(\mathbf{X}_{j, i}\right)=$ $g\left(\mathbf{X}_{j, i}\right)$, where $g$ is as defined in the statement of the theorem. From Equation (2), we can write

$\mathbf{Y}_{j, i}(\mathbf{X})=-\frac{\Psi\left(0 ; k_{j, i}\right)}{\Psi^{-1}\left(0 ; k_{j, i}\right)}\left[\mathbf{X}-\mathbf{X}_{j, i-1}\right]+\mathbf{Y}_{j, i-1}$ for $\mathbf{X} \in\left[\mathbf{X}_{j, i-1}, \mathbf{X}_{j, i}\right]$.

We choose $k_{j, i}$ so that each function $\mathbf{Y}_{j, i}(\mathbf{X})$ is a chord connecting the points $\left(\mathbf{X}_{j, i-1}, g\left(\mathbf{X}_{j, i-1}\right)\right)$ and $\left(\mathbf{X}_{j, i}, g\left(\mathbf{X}_{j, i}\right)\right)$. Hence,

$$
-\frac{\Psi\left(0 ; k_{j, i}\right)}{\Psi^{-1}\left(0 ; k_{j, i}\right)}=\frac{g\left(\mathbf{X}_{j, i}\right)-g\left(\mathbf{X}_{j, i-1}\right)}{\mathbf{X}_{j, i}-\mathbf{X}_{j, i-1}} .
$$

Such a $k_{j, i}$ exists because $-\infty<g^{\prime}(\mathbf{X})<0$ and $\Psi\left(0 ; k_{j, i}\right) /$ $\Psi^{-1}\left(0 ; k_{j, i}\right)$ is continuous in $k$, and $\left|\Psi\left(0 ; k_{j, i}\right) / \Psi^{-1}\left(0 ; k_{j, i}\right)\right|>$ $\left|\Psi\left(0 ; k_{j, i+1}\right) / \Psi^{-1}\left(0 ; k_{j, i+1}\right)\right|$. Therefore, we can choose a range of $k_{i} \mathrm{~s}$ sufficiently large to match any slope $\left(g\left(\mathbf{X}_{j, i}\right)-g\left(\mathbf{X}_{j, i-1}\right)\right) /\left(\mathbf{X}_{j, i}-\mathbf{X}_{j, i-1}\right)$ for all $\mathbf{X} \in\left[0, \mathbf{X}_{\max }\right]$. That is, there exist a range of $k_{j, i}$ s sufficiently large (small) to make the slope $-\Psi\left(0 ; k_{j, i}\right) / \Psi^{-1}\left(0 ; k_{j, i}\right)$ as shallow (steep) as needed.

Substituting into $\mathbf{Y}_{j, i}(\mathbf{X})$ gives

$$
\begin{array}{r}
\mathbf{Y}_{j, i}(\mathbf{X})=\left(\frac{g\left(\mathbf{X}_{j, i}\right)-g\left(\mathbf{X}_{j, i-1}\right)}{\mathbf{X}_{j, i}-\mathbf{X}_{j, i-1}}\right)\left[\mathbf{X}-\mathbf{X}_{j, i-1}\right]+g\left(\mathbf{X}_{j, i-1}\right) \\
\text { for } \mathbf{X} \in\left[\mathbf{X}_{j, i-1}, \mathbf{X}_{j, i}\right] .
\end{array}
$$

By the continuity of $g(\mathbf{X})$, for every $\epsilon>0$, there exists a $\delta>0$ such that if $\left|\mathbf{X}_{i, i-1}-\mathbf{X}_{j, i}\right|<\delta$ for all $i$, then $\mid g\left(\mathbf{X}_{i, i-1}\right)-$ $g\left(\mathbf{X}_{j, i}\right) \mid<\epsilon / 2$ for all $i$. Also, there exists a sufficiently fine partition $\mathbf{P}_{j^{*}}$ such that $\left|\mathbf{X}_{j^{*}, i}-\mathbf{X}_{j^{*}, i-1}\right| \leq \delta$ for all $i$ and for all $j>j^{*}$ a partition $\mathbf{P}_{j}$ will be such that $\left|\mathbf{X}_{j, i}-\mathbf{X}_{j, i-1}\right| \leq \delta$. 
For $\epsilon>0$, choose a partition $j>j^{*}$ so that $\left|\mathbf{X}_{j, i-1}-\mathbf{X}_{j, i}\right|<\delta$. For any $\mathbf{X} \in\left[0, \mathbf{X}_{\max }\right]$, without loss of generality, let $\mathbf{X} \in\left[\mathbf{X}_{j, i-1}, \mathbf{X}_{j, i}\right]$. Then we have

$$
\begin{aligned}
& \left|\mathbf{Y}_{j, i}(\mathbf{X})-g(\mathbf{X})\right| \\
& \quad=\left|\left(\frac{g\left(\mathbf{X}_{j, i}\right)-g\left(\mathbf{X}_{j, i-1}\right)}{\mathbf{X}_{j, i}-\mathbf{X}_{j, i-1}}\right)\left[\mathbf{X}-\mathbf{X}_{j, i-1}\right]+\left(g\left(\mathbf{X}_{j, i-1}\right)-g(\mathbf{X})\right)\right| \\
& \quad \leq\left|\left(\frac{\mathbf{X}-\mathbf{X}_{j, i-1}}{\mathbf{X}_{j, i}-\mathbf{X}_{j, i-1}}\right)\left[g\left(\mathbf{X}_{j, i}\right)-g\left(\mathbf{X}_{j, i-1}\right)\right]\right|+\left|\left(g\left(\mathbf{X}_{j, i-1}\right)-g(\mathbf{X})\right)\right| \\
& \quad \leq\left|g\left(\mathbf{X}_{j, i}\right)-g\left(\mathbf{X}_{j, i-1}\right)\right|+\left|\left(g\left(\mathbf{X}_{j, i-1}\right)-g(\mathbf{X})\right)\right| \leq \frac{\epsilon}{2}+\frac{\epsilon}{2}=\epsilon .
\end{aligned}
$$

This is true for any $\mathbf{X} \in\left[0, \mathbf{X}_{\max }\right]$ indicating uniform convergence. Hence, a risk-averse aggregate economy can be formed from an economy of individual risk-seeking agents with the wealth distribution associated with the partition $\mathbf{P}_{j}$ for $j>j^{*}$.

\section{References}

Appelbaum E, Katz E (1981) Market constraints as a rationale for the Friedman-Savage utility function. J. Political Econom. 89(4):819-825.

Atkeson A, Lucas RE Jr (1992) On efficient distribution with private information. Rev. Econom. Stud. 59(3):427-453.

Aumann RJ (1964) Markets with a continuum of traders. Econometrica 32(1-2):39-50.

Bakshi G, Chen Z (1996) The spirit of capitalism and stock-market prices. Amer. Econom. Rev. 86(1):133-157.

Bakshi G, Wu L (2010) The behavior of risk and market prices of risk over the Nasdaq bubble period. Management Sci. 56(12):2251-2264.

Baron D (1974) Information, investment behavior, and efficient portfolios. J. Financial Quant. Anal. 9(4):555-566.

Bawa VS (1977) Mathematical programming of admissible portfolios. Management Sci. 23(7):779-785.

Becker G (1962) Irrational behavior and economic theory. J. Political Econom. 70(1):1-13.

Blundell R, Stoker TM (2005) Heterogeneity and aggregation. J. Econom. Literature 43(2):347-391.

Bordley RF (1995) Possible convexity of the indirect utility function due to nonlinear budget constraints. Econom. Lett. 49(1):19-25.

Bossaerts P (2002) The Paradox of Asset Pricing (Princeton University Press, Princeton, NJ).

Chiappori PA, Ekeland I (1999) Aggregation and market demand: An exterior differential calculus viewpoint. Econometrica 67(6):1435-1457.

Constantinides GM (2002) Rational asset prices. J. Finance 57(4):1567-1591.

Constantinides GM, Duffie D (1996) Asset pricing with heterogeneous consumers. J. Political Econom. 104(2):219-240.

Coval JD, Shumway T (2005) Do behavioral biases affect prices? J. Finance 60(1):983-1009.

Eisenberg E (1961) Aggregation of utility functions. Management Sci. 7(4):337-350.

Esteban J, Ray D (2006) Inequality, lobbying, and resource allocation. Amer. Econom. Rev. 96(1):257-279.

Florentsen B, Rydqvist K (2002) Ex-day behavior when investors and professional traders assume reverse roles: The case of Danish lottery bonds. J. Financial Intermediation 11(2):152-175.

Foellmi R, Zweimuller J (2006) Income distribution and demandinduced innovations. Rev. Econom. Stud. 73(4):941-960.

Friedman M, Savage LJ (1948) The utility analysis of choices involving risk. J. Political Econom. 56(4):279-304.
Geanakoplos JD, Polemarchakis HM (1980) On the disaggregation of excess demand functions. Econometrica 48(2):315-331.

Gode D, Sunder S (1993) Allocative efficiency of markets with zerointelligence traders: Market as a partial substitute for individual rationality. J. Political Econom. 101(1):119-137.

Gode DK, Sunder S (1997) What makes markets allocationally efficient? Quart. J. Econom. 112(2):603-630.

Goetzmann WN, Massa M (2002) Daily momentum and contrarian behavior of index fund investors. J. Financial Quant. Anal. 37(3):375-389.

Gollier C (2001) Wealth inequality and asset pricing. Rev. Econom. Stud. 68(1):181-203.

Gottardi P, Hens T (1999) Disaggregation of excess demand and comparative statics with incomplete markets and nominal assets. Econom. Theory 13(2):287-308.

Green RC, Rydqvist K (1997) The valuation of non-systematic risks and the pricing of Swedish lottery bonds. Rev. Financial Stud. 10(2):447-480.

Gregory N (1980) Relative wealth and risk taking: A short note on the Friedman-Savage utility function. J. Political Econom. 88(6):1226-1230.

Hakansson NH (1970) Friedman-Savage utility functions consistent with risk aversion. Quart. J. Econom. 84(3):472-487.

Heaton J, Lucas D (1992) The effects of incomplete insurance markets and trading costs in a consumption-based asset pricing model J. Econom. Dynam. Control 16(3-4):601-620.

Hellwig MF (1980) On the aggregation of information in competitive markets. J. Econom. Theory 22(3):477-498.

Hellwig MF (1982) Rational expectations equilibrium with conditioning on past prices: A mean-variance example. J. Econom. Theory 26(2):279-312.

Hirshleifer D (2001) Investor psychology and asset pricing. J. Finance 56(4):1533-1597.

Jackwerth JC (2000) Recovering risk aversion from option prices and realized returns. Rev. Financial Stud. 13(2):433-451.

Jarrow RA (1988) Preferences, continuity, and the arbitrage pricing theory. Rev. Financial Stud. 1(2):159-172.

Jarrow R, Zhao F (2006) Downside loss aversion and portfolio management. Management Sci. 52(4):558-566.

Kahneman D, Tversky A (1979) Prospect theory of decisions under risk. Econometrica 47(2):263-291.

Kirman AP (1992) Whom or what does the representative individual represent? J. Econom. Perspectives 6(2):117-136.

Kliger D, Levy O (2002) Risk preferences heterogeneity: Evidence from asset markets. Eur. Finance Rev. 6(3):277-290.

Levy M, Levy H (2001) Testing for risk aversion: A stochastic dominance approach. Econom. Lett. 71(2):233-240.

Levy M, Levy H (2002) Prospect theory: Much ado about nothing? Management Sci. 48(10):1334-1349.

Lippman SA, McCall JJ, Winston WL (1980) Constant absolute risk aversion, bankruptcy, and wealth-dependent decisions. J. Bus. 53(3):285-296.

Markowitz H (1952) The utility of wealth. J. Political Econom. 60(2):151-158.

McCardle KF, Winkler RL (1992) Repeated gambles, learning, and risk aversion. Management Sci. 38(6):807-818.

Postlewaite A, Samuelson L, Silverman D (2008) Consumption commitments and employment contracts Rev. Econom. Stud. 75(2):559-578.

Robson AJ (1992) Status, the distribution of wealth, private and social attitudes toward risk. Econometrica 60(4):837-857.

Rosenberg JV, Engle RF (2002) Empirical pricing kernels. J. Financial Econom. 64(3):341-372.

Shefrin H (2005) A Behavioral Approach to Asset Pricing (Elsevier Academic Press, Burlington, MA).

Shefrin H, Statman M (2000) Behavioral portfolio theory. J. Financial Quant. Anal. 35(2):127-151. 